Marianna Csörnyei* Eötvös University, Department of Analysis, Múzeum Krt. 6-8, H-1088 Budapest, Hungary, e-mail: csornyei@cs.elte.hu

\title{
ON THE AFFINE SHARPNESS OF HEART'S DENSITY THEOREM
}

\begin{abstract}
Let $\left\{I_{n}\right\}_{n=1}^{\infty}$ be a sequence of pairwise disjoint intervals tending to 0 from the right, such that putting $I_{n}=\left(a_{n}, b_{n}\right)$ we have $\lim \inf \frac{\left|I_{n}\right|}{b_{n}}=0$. We prove that for every Cantor set $C$ there exists some set $X$ of positive measure, such that for a.e. $x \in X$ there exists a $c \in C$ for which $\left(x+c I_{n}\right) \cap X=\emptyset$ for infinitely many $n$.
\end{abstract}

V. Aversa and D. Preiss proved in [1] that there exists a sequence of pairwise disjoint positive intervals $\left\{I_{n}\right\}_{n=1}^{\infty}$ tending to 0 , such that putting $I_{n}=\left(a_{n}, b_{n}\right)$ we have

$$
\liminf \frac{\left|I_{n}\right|}{b_{n}}=0,
$$

but the differentiation system given by the translations of this system of intervals has the density property; that is, for every measurable set $X$ we have

$$
\left.\frac{\lambda\left(\left(x+I_{n}\right) \cap X\right)}{\left|I_{n}\right|} \rightarrow 1 \quad \text { a.e. } x \in X\right) .
$$

In the same paper it is proved that the differentiation system given by the affine images of this system has the density property if and only if (1) does not hold. Actually their proof shows that for every sequence of pairwise disjoint positive intervals tending to 0 for which (1) holds, there exists some set $X$ of positive measure, such that for a.e. $x \in X$ there exists a $c \in(0,1)$ for which $\left(x+c I_{n}\right) \cap X=\emptyset$ for infinitely many $n$.

In this paper, applying entirely different methods, we prove that the same statement holds even if the multipliers $c$ are taken only from a given Cantor set, rather than from $(0,1)$.

\footnotetext{
Mathematical Reviews subject classification: 28A75, 28A15

Received by the editors October 6, 1997

* Research supported by Grants FKFP 0189/1997 and Hungarian National Foundation for Scientific Research Grant No. T019476.
} 
Remark. This implies immediately that if the multipliers $c$ are taken from a Borel set $B$, then the statement holds if and only if $B$ is uncountable.

D. Borwein and S. Z. Ditor proved in [2] that there exists a set $X$ of positive measure and a sequence $d_{n}$ of positive numbers tending to 0 such that $x+d_{n} \notin X$ for infinitely many $n$.

A stronger result is proved in [3]. It is proved that for a sequence of pairwise disjoint positive intervals $\left\{I_{n}\right\}_{n=1}^{\infty}=\left\{I_{1}^{1}, I_{2}^{1}, \ldots, I_{n_{1}}^{1}, I_{1}^{2}, I_{2}^{2}, \ldots, I_{n_{2}}^{2}, \ldots\right\}$ tending to 0 , where $I_{1}^{j}, \ldots, I_{n_{j}}^{j}$ are of the same length and $\left\{n_{j}\right\}_{j=1}^{\infty}$ is not bounded, there exists a Cantor set $C$ of positive measure such that for a.e. $x \in C$ the interval $x+I_{n}$ is disjoint from $C$ for infinitely many $n$. As a generalization of this result now we prove the following lemma.

Lemma. Let $\left\{I_{n_{1} n_{2} \ldots n_{k+1}}: n_{1}=1,1 \leq n_{k+1} \leq m_{n_{1} n_{2} \ldots n_{k}}, k=0,1, \ldots\right\}$ be a system of open intervals, such that

(i) $I_{n_{1} n_{2} \ldots n_{k} j}\left(1 \leq j \leq m_{n_{1} n_{2} \ldots n_{k}}\right)$ are pairwise disjoint intervals of the same length;

(ii) in every sequence $I_{n_{1}}, I_{n_{1} n_{2}}, I_{n_{1} n_{2} n_{3}}, \ldots$ the intervals are pairwise disjoint and with lengths that tend to 0 ;

(iii) For every interval $I_{n_{1} n_{2} \ldots n_{k}}$ we have $\sup _{l_{1} l_{2} \ldots} m_{n_{1} n_{2} \ldots n_{k} l_{1} l_{2} \ldots l_{r}}=\infty$.

Then there exists a Cantor set $C$ of positive measure such that for a.e. $x \in C$ there exists a sequence $n_{1}, n_{2}, \ldots$ such that for infinitely many $k$ the intervals $x+I_{n_{1} n_{2} \ldots n_{k}}$ are disjoint from $C$.

Proof. We construct our Cantor set $C$ by a perfect scheme; that is, $C=\cap C_{n}$, where $C_{n}$ is the union of non-overlapping closed intervals each "associated to" one of the intervals $I_{n_{1} n_{2} \ldots n_{k}}$ of the same length, and $C_{1} \supset C_{2} \supset C_{3} \supset \ldots$.

For ease of notation we put $l_{n_{1} n_{2} \ldots n_{k}} \stackrel{\text { def }}{=}\left|I_{n_{1} n_{2} \ldots n_{k}}\right|$, and let $k_{n_{1} n_{2} \ldots n_{k}}$ be the least positive integer for which $\left(0, k_{n_{1} n_{2} \ldots n_{k}} \cdot l_{n_{1} n_{2} \ldots n_{k} j}\right)$ covers the union of intervals $I_{n_{1} n_{2} \ldots n_{k} j}\left(1 \leq j \leq m_{n_{1} n_{2} \ldots n_{k}}\right)$.

Let $C_{1}=\left[0, l_{1}\right]$, and we say that this interval is associated to $I_{1}$. Suppose we have already defined $C_{n-1}$, and let $J$ be one of its intervals, associated to the interval $I_{n_{1} n_{2} \ldots n_{k}}$. We choose an interval $I_{n_{1} n_{2} \ldots n_{k} l_{1} l_{2} \ldots l_{r}}$, for which putting $\bar{n} \stackrel{\text { def }}{=} n_{1} n_{2} \ldots n_{k}, \bar{l} \stackrel{\text { def }}{=} n_{1} n_{2} \ldots n_{k} l_{1} l_{2} \ldots l_{r}$ and $l^{*} \stackrel{\text { def }}{=} l_{\bar{l} j}$ we have

$$
\frac{k_{\bar{l}} l^{*}}{l_{\bar{n}}}<\frac{1}{n^{2}}
$$

and $m_{\bar{l}}>n^{2}$. This implies $k_{\bar{l}}>n^{2}$. 
Let $F$ be the set of intervals $\left((i-1) l^{*}, i l^{*}\right)$ where $i$ is a positive integer and this interval intersects some of the intervals $I_{\bar{l} j}$. Now, $F$ has at least $n^{2}$ elements. We choose $n$ elements, say with right endpoints $i_{1} l^{*}, \ldots, i_{n} l^{*}$. Let $G \stackrel{\text { def }}{=}\left\{i_{1}, \ldots, i_{n}\right\}$, and by the greedy algorithm we choose integers $0<$ $m_{1}, \ldots, m_{q} \leq k_{\bar{l}}$ for which the sets $G-m_{i}$ are pairwise disjoint (that is, we may choose $m_{1}=1$, let $m_{2}$ be the least integer for which $G-m_{1}$ and $G-m_{2}$ are disjoint, etc., etc.) Each set $G-m_{i}$ consists of $n$ intervals. Thus $\bigcup_{i=1}^{q}\left(G-m_{i}\right)$ intersects $G-m$ for at most $q n^{2}$ different values of $m$. We stop the algorithm when $\frac{k_{\bar{l}}}{n^{2}} \leq q \leq \frac{k_{\bar{l}}}{n^{2}}+1$. (We know $k_{\bar{l}}>n^{2}$ ).

Let

$$
A \stackrel{\text { def }}{=} \bigcup_{i=m_{p}-2, m_{p}-1, m_{p}, m_{p}+1,1 \leq p \leq q}\left((i-1) l^{*}, i l^{*}\right),
$$

and let

$$
B \stackrel{\text { def }}{=} \bigcup_{i=m_{p}-i_{t}, 1 \leq p \leq q, 1 \leq t \leq n}\left[(i-1) l^{*}, i l^{*}\right] \backslash A .
$$

Now, for every $\left((i-1) l^{*}, i l^{*}\right) \subset B$ there exists an $i_{t}$ such that

$$
x+\left(\left(i_{t}-2\right) l^{*},\left(i_{t}+1\right) l^{*}\right) \subset A ;
$$

that is, there is a $j$ such that $x+I_{\bar{l} j} \subset A$.

The measure of $A$ is "small", and the measure of $B$ is "large":

$$
\begin{gathered}
\frac{k_{l} l^{*}}{n^{2}} \leq q l^{*} \leq \lambda(A) \leq 4 q l^{*} \leq 8 \frac{k_{\bar{l}} l^{*}}{n^{2}}, \\
\lambda(B) \geq n q l^{*}-\lambda(A) \geq \frac{k_{l} l^{*}}{n}-8 \frac{k_{\bar{l}} l^{*}}{n^{2}},
\end{gathered}
$$

and $A \subset\left(-2 l^{*},\left(k_{\bar{l}}+1\right) l^{*}\right) \subset\left(-2 k_{\bar{l}} l^{*}, 2 k_{\bar{l}} l^{*}\right), B \subset\left[-k_{\bar{l}} l^{*}, k_{\bar{l}} l^{*}\right] \subset\left(-2 k_{\bar{l}} l^{*}, 2 k_{\bar{l}} l^{*}\right)$.

Now we construct $C_{n}$. For the interval $J=[a, b]$ associated to $I_{n_{1} n_{2} \ldots n_{k}}$ we consider the subintervals $\left[a+(4 s-4) k_{l} l^{*}, a+4 s k_{\bar{l}} l^{*}\right](s=1,2, \ldots)$, and we delete the remainder of the interval $\left(a+4 s k_{l} l^{*}, b\right)$ of length less than $4 k_{l} l^{*}$, and we delete the sets $a+(4 s-2) k_{\bar{l}} l^{*}+A \subset a+\left[(4 s-4) k_{\bar{l}} l^{*}, a+4 s k_{\bar{l}} l^{*}\right]$, and possible isolated points. For every interval $I=a+(4 s-2) k_{l} l^{*}+\left((i-1) l^{*}, i l^{*}\right)$, where $\left((i-1) l^{*}, i l^{*}\right) \subset B$, there is an interval $I_{\bar{l} j}$ for which $x+I_{\bar{l} j}$ lies in the deleted set $x+(4 s-2) k_{l} l^{*}+A$. The interval $a+(4 s-2) k_{l} l^{*}+\left[(i-1) l^{*}, i l^{*}\right]$ is said to be associated to one of these intervals $I_{\bar{l} j}$, and the other non-deleted intervals of form $a+(4 s-2) k_{l} l^{*}+\left[(i-1) l^{*}, i l^{*}\right]$ are said to be associated to an interval $I_{\bar{l} j}$ where $j$ is chosen arbitrarily. This process yields a Cantor set $C$.

According to (2) and (3) the set $C$ constructed is of positive measure, and according to (4) the set of points of $C$ contained in a set of the form $a+(4 s-2) k_{i} l^{*}+B$ only finitely often is of measure 0 . 
Theorem. For a sequence of pairwise disjoint intervals $\left\{J_{n}\right\}_{n=1}^{\infty}$ and a Cantor set $E$ condition (1) implies that there exists a Cantor set $C$ of positive measure, such that for a.e. $x \in C$ there exists a $c \in E$ for which $x+c J_{n}$ is disjoint from $C$ for infinitely many $n$.

Proof. Let $J_{n}=\left(a_{n}, b_{n}\right)$ satisfy (1); that is, $\liminf \frac{b_{n}-a_{n}}{b_{n}}=0$. Let $E=\cap E_{n}$ be a Cantor set, where $E_{1} \supset E_{2} \supset \ldots$ are the closed sets of a perfect scheme defining $E$, each is the union of finitely many closed intervals. We construct a system of intervals $I_{n_{1} n_{2} \ldots n_{k}}$ satisfying the conditions of the lemma, together with another system of closed intervals $L_{n_{1} n_{2} \ldots n_{k}} \subset E_{k}$ for which $L_{n_{1} n_{2} \ldots n_{k}} \supset L_{n_{1} n_{2} \ldots n_{k} n_{k+1}} \supset \ldots, \operatorname{int} L_{n_{1} n_{2} \ldots n_{k}} \cap E \neq \emptyset$, and for every $n_{1} n_{2} \ldots n_{k}$ there exists an interval $J_{n}$ of our sequence, such that for every $c \in L_{n_{1} n_{2} \ldots n_{k}}$ we have $c J_{n} \subset I_{n_{1} n_{2} \ldots n_{k}}$. Having these systems of intervals defined the theorem easily follows because by the Lemma there exists a Cantor set $C$ of positive measure such that for a.e. $x \in C$ there exist a branch $I_{n_{1}}, I_{n_{1} n_{2}} \ldots$ and infinitely many $k$ for which $C \cap\left(x+I_{n_{1} n_{2} \ldots n_{k}}\right)=\emptyset$. Hence for $c \in \bigcap_{k} L_{n_{1} n_{2} \ldots n_{k}} \subset \bigcap_{k} E_{k}=E$ we have $C \cap\left(x+c J_{n}\right)=\emptyset$ for infinitely many $n$.

Let $L_{1}=\left[c_{1}, d_{1}\right] \subset E_{1}$ such that $\left(c_{1}, d_{1}\right) \cap E \neq \emptyset$, and put $I_{1} \stackrel{\text { def }}{=}\left(c_{1} a_{1}, d_{1} b_{1}\right)$. Suppose we have already defined the intervals $I_{n_{1} n_{2} \ldots n_{k}}$ and $L_{n_{1} n_{2} \ldots n_{k}}=$ $[c, d]$, where $(c, d) \cap E \neq \emptyset$.

We choose $\delta>0$ and also points $\lambda^{1}, \lambda^{2}, \ldots, \lambda^{k} \in(c, d) \cap E$ such that $\lambda^{1}<$ $\lambda^{2}<\ldots<\lambda^{k},\left(\lambda^{j}-\delta, \lambda^{j}\right) \subset E_{k}$, and for each $\lambda^{j}$ and for every $\varepsilon>0$ we have $\left(\lambda^{j}-\varepsilon, \lambda^{j}\right) \cap E \neq \emptyset$. We can choose $J_{n}=\left(a_{n}, b_{n}\right)=(a, b)$ such that $(0, d b) \cap I_{n_{1} n_{2} \ldots n_{k}}=\emptyset$, and putting

$$
b^{j} \stackrel{\text { def }}{=} \lambda^{j} b, a^{j} \stackrel{\text { def }}{=} \lambda^{j} b-d(b-a)
$$

the intervals $\left[a^{1}, b^{1}\right],\left[a^{2}, b^{2}\right], \ldots,\left[a^{k}, b^{k}\right]$ are pairwise disjoint subintervals of $(c a, d b)$ of length $d(b-a)$, and $\frac{a^{j}}{a}>\lambda^{j}-\delta$. Indeed, we observe that the two conditions $c a<a^{1}, b^{j}<a^{j+1}$ and $\frac{a^{j}}{a}>\lambda^{j}-\delta$ hold if and only if $\frac{\left|J_{n}\right|}{b_{n}}<\min \left(\frac{\lambda^{1}-c}{d-c}, \frac{\lambda^{j+1}-\lambda^{j}}{d}, \frac{d-\lambda^{j}+\delta}{\delta}\right)$.

Let

$$
I_{n_{1} n_{2} \ldots n_{k} j} \stackrel{\text { def }}{=}\left[a^{j}, b^{j}\right]\left(j=1,2, \ldots, m_{n_{1} n_{2} \ldots n_{k}} \stackrel{\text { def }}{=} k\right)
$$

and

$$
L_{n_{1} n_{2} \ldots n_{k} j} \stackrel{\text { def }}{=}\left[\frac{a^{j}}{a}, \frac{b^{j}}{b}\right]
$$

We need

$$
c<\frac{a^{1}}{a}<\frac{b^{1}}{b}<\frac{a^{2}}{a}<\ldots<\frac{b^{k}}{b}<d .
$$


The inequalities $c<\frac{a^{1}}{a}$ and $\frac{b^{j}}{b}<d$ follow immediately from $c a<a^{1}$ and $\lambda^{j} \in(c, d)$. Since $\lambda^{j}=\frac{b_{j}}{b}<d$, the length of the interval $\frac{b^{j}}{b}[a, b]=\left[\frac{b^{j}}{b} a, b^{j}\right]$ is less than $d(b-a)$, that is, $a^{j}<\frac{b^{j}}{b} a$. Finally, $a<b$ and $b^{j-1}<a^{j}$ implies $\frac{b^{j-1}}{b}<\frac{a^{j}}{a}$.

Now, $L_{n_{1} n_{2} \ldots n_{k} j}=\left[\frac{a^{j}}{a}, \lambda^{j}\right] \subset\left[\lambda^{j}-\delta, \lambda^{j}\right] \subset E_{k}$ and $\operatorname{int} L_{n_{1} n_{2} \ldots n_{k} j} \cap E \neq \emptyset$. From the definition of $L_{n_{1} n_{2} \ldots n_{k} j}$ it follows that, for every $c \in L_{n_{1} n_{2} \ldots n_{k} j}$ we have $c J_{n}=c(a, b) \subset I_{n_{1} n_{2} \ldots n_{k} j}$ and conditions (i)-(iii) are easily verified.

\section{References}

[1] V. Aversa, D. Preiss, Hearts density theorems, Real Analysis Exchange, 13 No 1 (1987-88), 28-32.

[2] D. Borwein, S. Z. Ditor, Translates of sequences in sets of positive measure, Canad. Math. Bull. 21 (1978), 497-498.

[3] M. Csörnyei, Density theorems revisited, Acta Sci. Math. (Szeged), 64 (1998), 59-65. 
